\title{
Preservation of Dietary Triglycerides in the Secondary Particles of Alimentary Lipemia *
}

\author{
ENOCH GORDIS $\dagger$ \\ (From the Rockefeller Institute, New York, N.Y.)
}

Transport of triglycerides from intestine to blood and from blood to adipose and other organs involves several steps. In the intestine, pancreatic lipase partially hydrolyzes the dietary fat. During absorption, fatty acids and lower glycerides are reesterified and discharged into the lymph as chylomicrons. From lymph they pass to blood, and then to various tissues, notably liver and adipose tissue (1-3). During alimentary lipemia, the fat particles of blood plasma are heterogeneous $(4,5)$. In addition to chylomicron-type particles ("primary" particles), a second group of particles can be demonstrated. These particles, called "secondary" particles, behave differently during electrophoresis and during flocculation by polyvinylpyrrolidone (PVP). The fatty acid composition of the triglycerides contained in the secondary particles varies during alimentary lipemia. Two hours after a fatty meal, the composition resembles that of depot fat; at 9 hours, it resembles the composition of fed fat. It is evident that dietary lipids enter the secondary particles. This can be seen clearly after feeding corn oil, a fat rich in linoleic acid. The ratio of linoleic acid to oleic acid in corn oil is 1.7 . In a typical study of alimentary lipemia produced by feeding corn oil (4), the value of this ratio in the secondary particles at 2 hours was 0.37 , and at 9 hours, 1.37.

A mixture of triglycerides containing both dietary and endogenous fatty acids might be formed either by re-esterifying a mixture of endogenous and dietary acids after hydrolysis of dietary fat, or by incorporating into the same particles intact unhydrolyzed dietary triglycerides and endogenous glycerides. To determine which of these processes accounts for the composition of the secondary particles, methods to separate triglyceride molecules must be used. In the experiments reported here, the fed fat contained a characteristic group

* Submitted for publication March 11, 1965; accepted May 12, 1965.

Supported by U. S. Public Health Service grant AM 03963-05.

$\dagger$ Address requests for reprints to Dr. Enoch Gordis, Rockefeller Institute, New York, N. Y. 10021. of triglyceride molecules whose survival could be followed during fat transport. The evidence indicates that a considerable number of triglyceride molecules having the structure of dietary fat are incorporated into the secondary particles.

\section{Methods}

To resolve triglycerides into fractions containing molecules with as many as six double bonds, the method of Kaufmann and Wessels (6) was used with but slight modification. This method is an extension of the original procedure of Barrett, Dallas, and Padley (7), who were the first to separate triglycerides according to their degree of unsaturation by thin-layer chromatography on silicic acid impregnated with silver nitrate. A slurry containing $70 \mathrm{~g}$ of silica gel $\mathrm{G}$ and $140 \mathrm{ml}$ of $12 \frac{1}{2} \%$ aqueous silver nitrate was spread as a film about $700 \mu$ thick on two plates, $20 \mathrm{~cm} \times 40 \mathrm{~cm}$. The plates were kept in darkness overnight and then activated $2 \frac{1}{2}$ hours at $140^{\circ} \mathrm{C}$. Up to $50 \mathrm{mg}$ of triglyceride was applied in a line parallel to the short edge of the plate, and the fractions were separated by ascending chromatography in benzene-ethyl ether 85:15 vol/vol. The solvent front reached a level $2 \mathrm{~cm}$ below the top of the plate in about 5 hours. After spraying the plate with $0.3 \%$ dichlorofluorescein in methanol, the glyceride fractions appeared as yellow bands in ultraviolet light. The silicic acid of each band was then scraped off with a razor blade and packed into a small column at the bottom of which a small amount of activated silicic acid ${ }^{1}$ had been placed to trap the fluorescein. The glycerides were eluted from the silica gel with $30 \mathrm{ml}$ of ethyl ether. As an internal standard, equal volumes of a solution of arachidic acid in petroleum ether were added to all the eluates from a single chromatographic plate. The eluates were then extracted with water to remove $\mathrm{AgNO}_{3}$, dried with sodium sulfate, and evaporated. For gas-liquid chromatography of the triglyceride fatty acids, the lipid residues were transmethylated with $\mathrm{HCl}$-methanol by the method of Stoffel, Chu, and Ahrens (8).

For each fraction obtained from the plate, the fatty acid composition was determined. The ratio of triglyceride fatty acids to the arachidic acid standard was also determined in each fraction, and from the values of this ratio in all the fractions, the proportions of glycerides in the different fractions were calculated. Quantitative recovery of separated glycerides was checked as follows: A mixture of tripalmitin, triolein, and trilinolein was made from pure materials, and its composition was determined

1 Bio-Rad Laboratories, Richmond, Calif. 
TABLE I

Determination of proportions of triglycerides in standard mixture by quantitative elution after chromatography on $\mathrm{AgNO} \mathrm{g}_{3}$-silica gel $\mathrm{G}$

\begin{tabular}{|c|c|c|c|c|c|c|}
\hline & $\begin{array}{c}\text { Known } \\
\text { composition }\end{array}$ & Sample A & Sample B & Sample C & Sample D & Mean \pm SD \\
\hline & $\%$ & $\%$ & $\%$ & $\%$ & $\%$ & $\%$ \\
\hline Tripalmitin & 34.1 & 37.4 & 31.9 & 32.2 & 34.0 & $33.9 \pm 2.5$ \\
\hline Triolein & 14.2 & 15.9 & 14.8 & 14.3 & 15.2 & $15.1 \pm 1.7$ \\
\hline Trilinolein & 51.6 & 46.6 & 53.3 & 53.5 & 50.3 & $50.9 \pm 2.7$ \\
\hline
\end{tabular}

by gas-liquid chromatography of the fatty acid methyl esters produced by transesterifying a sample of the mixture. Four samples of this mixture were resolved into their component glycerides by thin-layer chromatography on $\mathrm{AgNO}_{3}$-silica gel $\mathrm{G}$, and the proportions of the glycer-

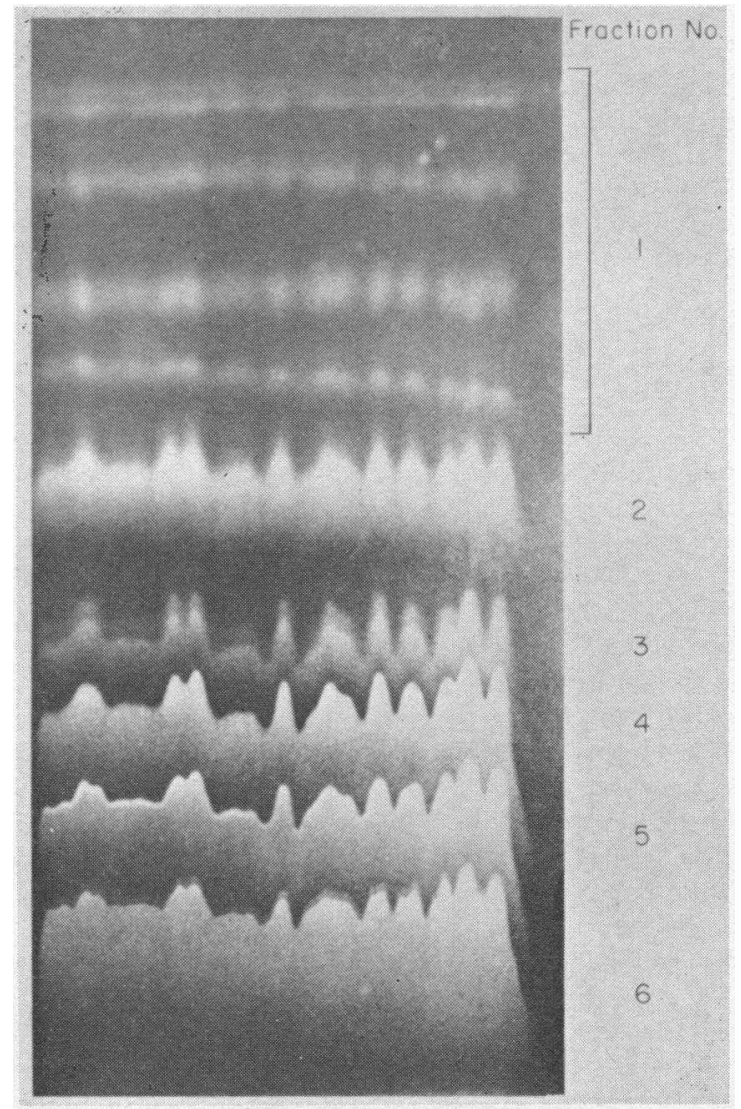

Fig. 1. Fractionation OF SAFFlower OIL TRIGLYCERIDES BY CHROMATOGRAPHY ON AGNOs-SILICA GEL G Load: approximately $30 \mathrm{mg}$. Solvent: benzene-ethyl ether $85: 15 \mathrm{vol} / \mathrm{vol}$. Photograph made in ultraviolet light after spraying plate with $0.3 \%$ dichlorofluorescein in methanol. Triglycerides are resolved according to their degree of unsaturation. Saturated glycerides move with the solvent front. Safflower oil contains 60\% [222] (fraction 6). The survival of this fraction was studied during fat transport. ides were determined with the internal standard method described above. The results are presented in Table I.

Primary and secondary particles may be obtained separately from lipemic blood plasma either by differential flocculation in gradient columns of polyvinylpyrrolidone (PVP) (4) or by electrophoresis on starch block (5). The particles analyzed in these experiments were obtained from postprandial blood by PVP gradient flocculation. Secondary particles were removed from the gradient tubes with a pipette and centrifuged upward into isotonic saline (D 1.006) in the SW-39 head of a Spinco model L ultracentrifuge at $3 \times 10^{6} g$-minute $(g$-minute $=$ gravitational field $\times$ time of centrifugation). The saline layers were removed, mixed with $20 \mathrm{vol}$ of chloroform-methanol $2: 1$ ( vol/vol), and filtered. Water ( $0.2 \mathrm{vol})$ was added, the phases were separated, and the chloroform phases were removed and evaporated. The lipid residues were dissolved in petroleum ether ( $\mathrm{bp} 30^{\circ}$ to $60^{\circ} \mathrm{C}$ ) and the triglycerides isolated from them by thin-layer chromatography on plates of unmodified silica gel $\mathrm{G}$ (solvent: petroleum ether-ethyl ether $90: 10 \mathrm{vol} / \mathrm{vol}$ ).

Gas-liquid chromatography of fatty acid methyl esters was performed on a 6-foot column packed with $17 \%$ ethylene glycol succinate on Chromosorb W. ${ }^{2}$ Effluents were detected with a strontium ${ }^{90}$ ionization detector, calibrated with appropriate methyl ester standard mixtures. ${ }^{2}$

Solvents were reagent grade, distilled before use.

Tripalmitin, triolein, trilinolein, and arachidic acid standards, $99 \%$ pure, were obtained commercially. ${ }^{2}$ The homogeneity of these materials was checked by thin-layer chromatography of the glycerides and by gas-liquid chromatography of the fatty acid methyl esters derived from these materials.

Silica gel G, containing $13 \%$ calcium sulfate, was obtained commercially. ${ }^{3}$ Silver nitrate crystals were of analytical reagent grade. Safflower oil was a commercial food product.4

The position of fatty acids on glycerol carbons was determined with the pancreatic lipase method of Mattson and Volpenhein (9), suitably scaled down for analysis of $10-\mathrm{mg}$ samples.

Nomenclature. Triglycerides can be classified according to the degree of unsaturation. This is convenient, since the classification corresponds to the physical separa-

2 Applied Science Laboratories, State College, Pa.

3 E. Merck and Co., Darmstadt, Germany.

4 Saff-O-Life, General Mills, Minneapolis, Minn. 


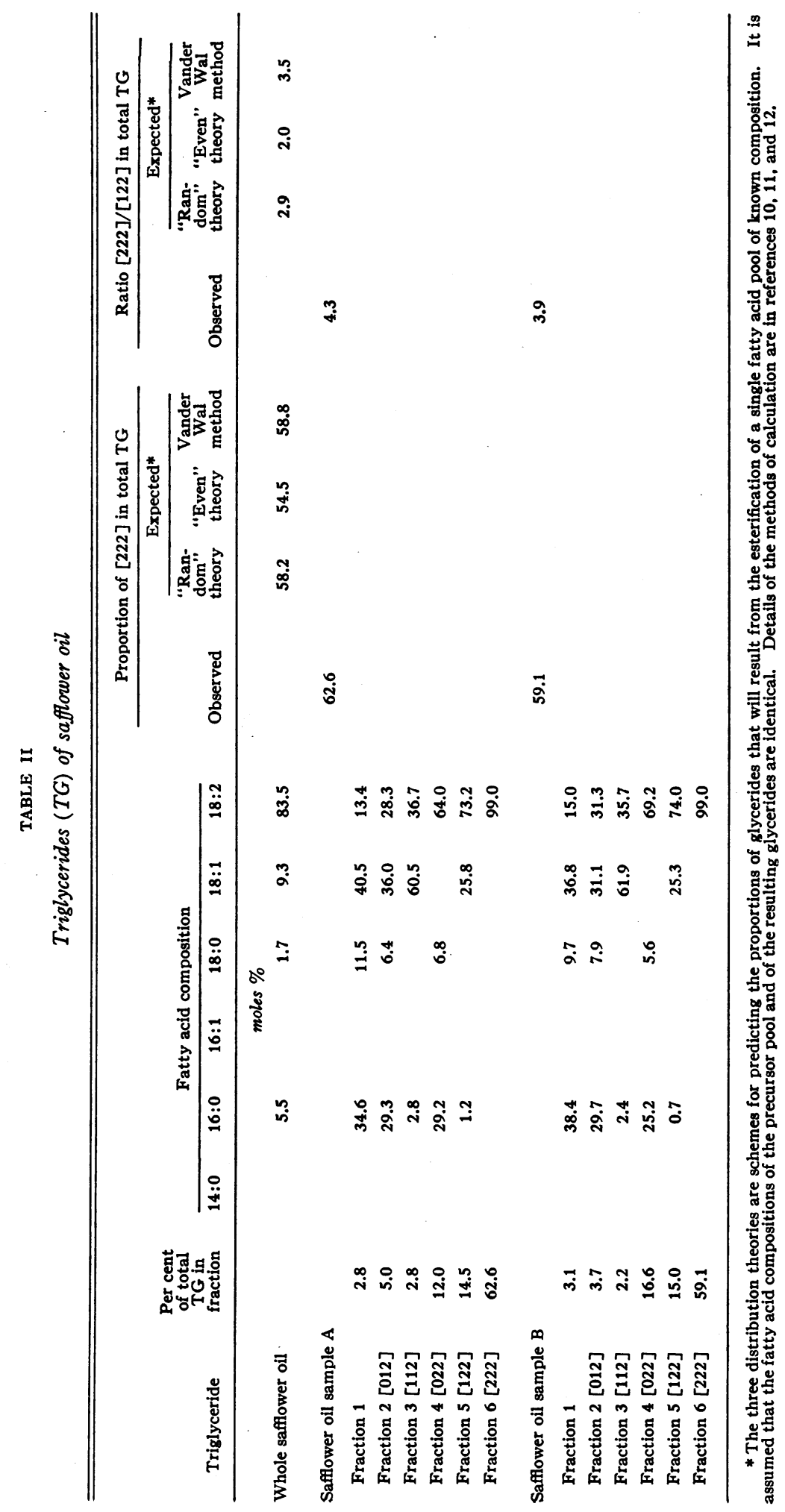


tion of triglycerides on the chromatography plate. Each triglyceride class is symbolized by a three-digit number enclosed in square brackets. Each digit represents the number of double bonds in one of the fatty acids of the molecule. Thus, trilinolein, containing six double bonds, is identified as [222]. When positional isomers occur in a group of triglycerides, the number in square brackets represents the group without regard to the position of the fatty acids. The digits are written in ascending order. To represent positional isomers within a group, upper bars are used. Thus, all the oleodilinoleins are symbolized by [122], but the isomers conventionally called 1-oleodilinolein and 2-oleodilinolein are symbolized by $\overline{122}$ and $\overline{212}$, respectively. For display of fatty acid compositions, each fatty acid is symbolized by two numbers separated by a colon. The first number is the number of carbons in the chain, the second the number of double bonds. In the present context, [222] can represent only trilinolein, since $18: 2$ is the only dienoic acid encountered. The monoenoic acids include 18:1 and some $16: 1$, and the saturated acids include $16: 0,18: 0$, and some $14: 0$.

\section{Results}

I. The glycerides of safflower oil. The triglycerides of a commercial safflower oil were isolated by preparative thin-layer chromatography on unmodified silica gel $\mathrm{G}$. Two samples of this triglyceride of about $30 \mathrm{mg}$ each were then analyzed by chromatography on thin layers of $\mathrm{AgNO}_{3}$-silica gel G. Nine bands were observed (Figure 1). The upper four bands contained less than $4 \%$ of the total sample and were pooled as one fraction. For each fraction, Table II lists the symbol for its glycerides, its proportion in the total sample, and its molar fatty acid composition. [222] is isolated as a pure molecular species, since $18: 2$ is the only dienoic acid present. [122] contains only $18: 1$ and $18: 2$, but both positional isomers, $\overline{122}$ and $\overline{212}$, are present. Triglycerides with four double bonds are separated into two groups. The [022] group contains only $\overline{022}$, but the saturated acid may be either $16: 0$ or $18: 0$. The [112] group contains both isomers, $\overline{112}$ and $\overline{121}$. These results are consistent with Mattson and Lutton's well-known finding (13) that saturated acids are almost completely excluded from the middle glycerol carbons of many unsaturated seed oils.

From the fatty acid composition of safflower oil, the expected proportions of [222] and of the ratio [222]/[122] have been calculated according to three theories of glyceride structure: the "random distribution" theory, the "even distribution" theory (10), and Vander Wal's theory (11), which al- lows for specific esterification of unsaturated acids at the middle glycerol carbon. The observed values are in fair agreement with Vander Wal's system. This theory, however, can be regarded as no more than a convenient estimate of the expected proportions of glycerides in a natural mixture. It does not predict all details of glyceride biosynthesis, since analysis of the proportion of isomers in a group such as [122] shows that the ratio of $\overline{122}$ to $\overline{212}$ is far in excess of the predicted values (14).

II. The glycerides of the secondary particles. Safflower oil, with its high content of $18: 2$, and its distinctively high content of [222] and [122], was chosen for the feeding experiments. It is suitable for two reasons. The body normally does not synthesize these highly unsaturated glycerides.

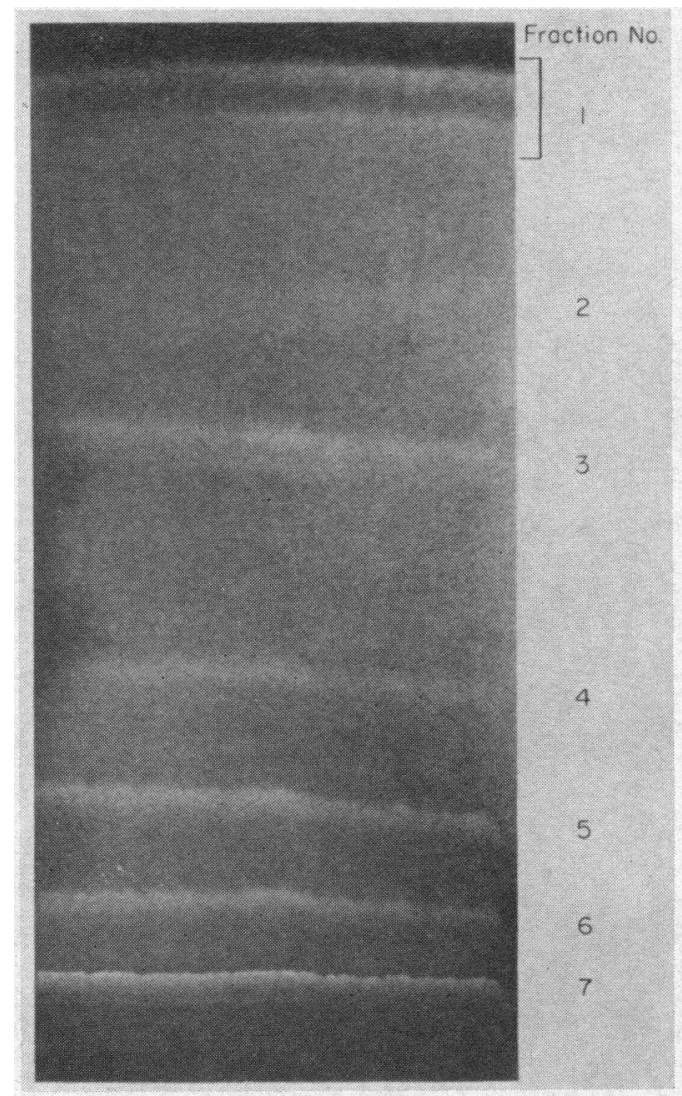

Fig. 2. Fractionation OF TRIGLYCERIDES OF SECONDARY PARTICLES FROM SUBJECTS FED SAFFLOWER OIL. Chromatography on $\mathrm{AgNO}_{3}$-silica gel G. Load: approximately $10 \mathrm{mg}$. Solvent: benzene-ethyl ether $85: 15 \mathrm{vol} / \mathrm{vol}$. The glyceride group [222] (fraction 7) predominates in safflower oil. It is seen to be a prominent component of secondary particle triglyceride also, 
TABLE III

Triglycerides of secondary particles

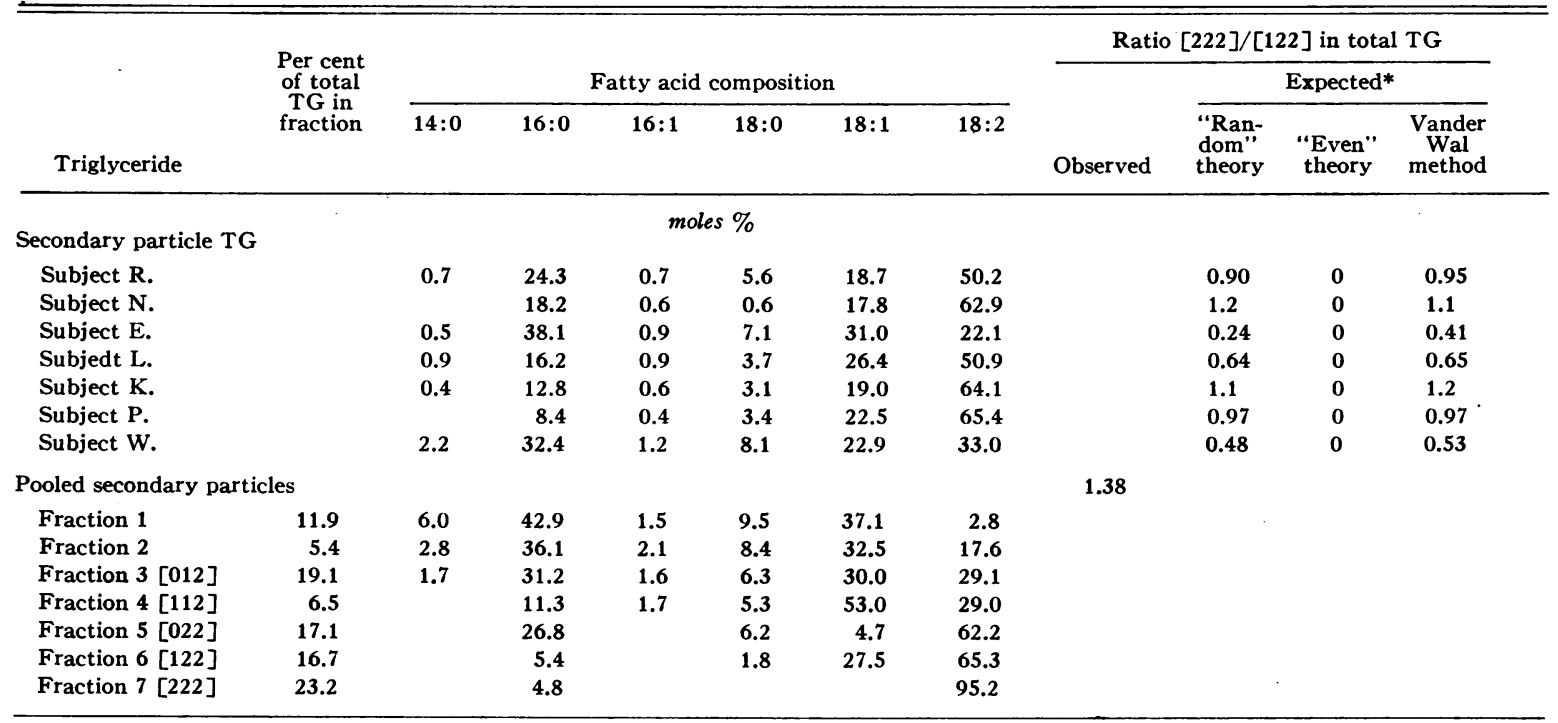

* See footnote to Table II.

In addition, hydrolysis and esterification in the intestine cannot significantly alter the proportions of the glycerides, since most of the fatty acids on the middle glycerol carbons are not hydrolyzed (15).

Seven normal fasting males were fed $200 \mathrm{~g}$ of safflower oil mixed with fat-free milk. Five hours later, venous blood was collected from each subject and prevented from clotting with Versene. Nineteen and two-tenths $\mathrm{ml}$ of plasma from each subject was fractionated with PVP-gradient tubes. Since each tube accommodates $0.8 \mathrm{ml}$ of plasma (4), 24 gradient tubes were prepared for each of the seven subjects. The plasmas were only moderately turbid, and there were not enough primary particles for analysis. The secondary particles were removed and their triglycerides isolated. The fatty acid composition of each triglyceride sample was determined. To have sufficient material for glyceride analysis, the seven samples were pooled. About $10 \mathrm{mg}$ of secondary particle triglyceride was then fractionated by chromatography on $\mathrm{AgNO}_{3}$ silica gel G. Figure 2 shows this chromatographic plate and the manner in which the fractions were numbered. Table III lists the molar fatty acid compositions of the individual secondary particle samples, the proportions of the glycerides in the pooled mixture, and the molar fatty acid compositions of the different fractions. The observed value of the ratio $[222] /[122]$ in the pooled sam- ple was 1.38. To determine whether this ratio could have resulted from the interesterification of the dietary and endogenous fatty acids, the expected value of this ratio was calculated from the molar fatty acid compositions of the individual samples. The right side of Table III lists the expected value of this ratio calculated on the basis of three theories of glyceride structure. Regardless of the method of calculation, the observed value of the ratio $[222] /[122]$ exceeded the expected value in every case.

\section{Discussion}

The excess of [222] in the pooled sample is most readily explained by the incorporation into secondary particles of intact triglycerides having the structure of dietary fat. An estimate of the maximal extent of this incorporation can be made. If all the [222] in the pooled sample came from the diet accompanied by as much of the other glycerides as are needed to complete the safflower oil pattern, dietary glycerides could account for $40 \%$ of the sample. The other, more saturated glycerides of the secondary particles are esterified from a blend of endogenous and dietary fatty acids.

The structure of the secondary particle triglyceride described in this report can be understood in the light of recent investigations of the origin of these particles. Bierman and Strandness have 
shown (16) that secondary particles can be formed within the circulating blood from primary particles without the intervention of the liver. When lymph was infused into hepatectomized, partially eviscerated dogs, secondary particles were produced. Secondary particles were also produced in vitro by incubating lymph with appropriate amounts of clear plasma. Bierman has proposed that secondary particles result from the interaction of primary particles with a soluble plasma lipoprotein. Plasma lipoproteins are synthesized by the liver. During alimentary lipemia, dietary fatty acids are incorporated into lipoprotein triglyceride as well as into particles $(17,18)$. The mechanism proposed by Bierman strongly suggests that the triglyceride of the secondary particle would consist of a mixture of molecules from two sources: primary particle triglyceride, containing molecules with the structure of dietary fat, and lipoprotein triglyceride, containing molecules produced in the liver by esterification of both dietary and endogenous fatty acids.

The extent to which triglyceride molecules survive in the body with their original glycerol and fatty acids attached to each other has been studied with fat labeled in both the glycerol and fatty acid moieties. Reiser, Williams, and Sorrels (19) fed tracer amounts of glyceryl- $\mathrm{C}^{\mathbf{1 4}}$-tripalmitate- $\mathrm{H}^{3}$ to rats. Three hours later, the isotope ratio $\mathrm{C}^{14} / \mathrm{H}^{3}$ in the triglycerides of blood and liver was one-half its original value. At 6 hours the ratio in plasma was still two-fifths of its initial value. The isotope ratio in individual lipoproteins was not studied. It may be objected that the labeled materials, although present in the same sample, may no longer have been esterified as tripalmitin. However, when the data of Reiser and associates are considered, together with the glyceride analysis of secondary particles reported here, the survival of dietary triglyceride molecules appears highly likely.

The study of Borgström and Jordan (20), however, appears inconsistent with this conclusion. These investigators injected intravenously doubly labeled chylomicrons obtained from rat lymph. The ratio of glycerol to fatty acid radioactivities in the neutral fat of plasma dropped to one-tenth its original value in 40 minutes. The experiments of Borgström and Jordan, however, differ in two respects from those reported here and from those of Reiser, Williams, and Sorrels. First, Borgström and Jordan pulse labeled the plasma compartment by a single injection of radioactive chylomicrons, whereas Reiser's study and the present one involved, in effect, the continuous infusion of chylomicrons into the circulation. In addition, the physical state of the material injected by Borgström and Jordan differed markedly from its physiological state. Lymph chylomicrons were injected, but they were "washed" by centrifugation into saline before use. It is uncertain if normal particles and particles deprived of their protein milieu by washing have the same fate during transport.

\section{Summary}

The structure of the triglycerides in the secondary particles of alimentary lipemia was investigated. Normal subjects ingested a fat containing a distinctive group of triglycerides. The secondary particles appearing in the blood contained a large proportion of these triglycerides. It is concluded that secondary particles contain triglycerides from two sources: triglycerides having the structure of dietary fat, and other triglycerides esterified from a mixed pool of endogenous and dietary fatty acids. These results are consistent with Bierman's recent proposal that secondary particles originate in the blood by instantaneous mixing of primary particles from the lymph and soluble lipoprotein without intervention by the liver.

\section{References}

1. Bradgon, J. H., and R. S. Gordon, Jr. Tissue distribution of $\mathrm{C}^{14}$ after the intravenous injection of labeled chylomicrons and unesterified fatty acids in the rat. J. clin. Invest. 1958, 37, 574.

2. Havel, R. J., and A. Goldfien. The role of the liver and of extrahepatic tissues in the transport and metabolism of fatty acids and triglycerides in the dog. J. Lipid Res. 1961, 2, 389.

3. Nestel, P. J., R. J. Havel, and A. Bezman. Sites of initial removal of chylomicron triglyceride fatty acids from the blood. J. clin. Invest. 1962, 41, 1915.

4. Gordis, E. Demonstration of two kinds of fat particles in alimentary lipemia with polyvinylpyrrolidone gradient columns. Proc. Soc. exp. Biol. (N. Y.) 1962, 110, 657.

5. Bierman, E. L., E. Gordis, and J. T. Hamlin III. Heterogeneity of fat particles in plasma during alimentary lipemia. J. clin. Invest. 1962, 41, 2254. 
6. Kaufmann, H. P., and H. Wessels. Die DünnschichtChromatographie auf dem Fettgebiet XIV: die Trennung der Triglyceride durch Kombination der Adsorptions-und der Umkehrphasen-Chromatographie. Fette-Seifen-Anstrichmittel 1964, 66, 81.

7. Barrett, C. B., M. S. J. Dallas, and F. B. Padley. The separation of glycerides by thin-layer chromatography on silica impregnated with silver nitrate. Chem. and Ind. 1962, 1050.

8. Stoffel, W., F. Chu, and E. H. Ahrens, Jr. Analysis of long-chain fatty acids by gas-liquid chromatography: micromethod for preparation of methyl esters. Analyt. Chem. 1959, 31, 307.

9. Mattson, F. H., and R. A. Volpenhein. The use of pancreatic lipase for determining the distribution of fatty acids in partial and complete glycerides. J. Lipid Res. 1961, 2, 58.

10. Hilditch, T. P. The Chemical Constitution of Natural Fats, 3rd ed. rev. New York, John Wiley, 1956 , p. 16.

11. Vander Wal, R. J. Calculation of the distribution of the saturated and unsaturated acyl groups in fats, from pancreatic lipase hydrolysis data. J. Amer. Oil Chemists' Soc. 1960, 37, 18.

12. Dutton, H. J., and C. R. Scholfield. Recent developments in the glyceride structure of vegetable oils in Progress in the Chemistry of Fats and Other Lipids. New York, Macmillan, 1963, p. 314.

13. Mattson, F. H., and E. S. Lutton. The specific distribution of fatty acids in the glycerides of animal and vegetable fats. J. biol. Chem. 1958, 233, 868.

14. Gordis, E. Unpublished observations.

15. Mattson, F. H., and R. A. Volpenhein. Rearrangement of glyceride fatty acids during digestion and absorption. J. biol. Chem. 1962, 237, 53.

16. Bierman, E. L., and D. E. Strandness. The mechanism of formation of secondary fat particles from lymph chylomicrons (abstract). J. clin. Invest. 1965, 44, 1028.

17. Bragdon, J. H., and A. Karmen. Effect of ingested fat on fatty acid composition of serum lipoproteins. J. Lipid Res. 1961, 2, 400.

18. Nichols, A. V., C. S. Rehnborg, F. T. Lindgren, and R. D. Wills. Effects of oil ingestion on lipoprotein fatty acids in man. J. Lipid Res. 1962, 3, 320.

19. Reiser, R., M. C. Williams, and M. F. Sorrels. The transport and dynamic state of exogenous glyceroland palmitic acid-labeled tripalmitin. J. Lipid Res. 1960, 1, 241.

20. Borgström, B., and P. Jordan. Metabolism of chylomicron glyceride as studied by $\mathrm{C}^{\mathbf{1 4}}$-glycerol- $\mathrm{C}^{14}$ palmitic acid labeled chylomicrons. Acta Soc. Med. upsalien. 1959, 64, 185. 